\title{
Relationship between the Northern Hemisphere Sea Ice Area and Global Temperature by Multifractal Analysis
}

\author{
Fumio Maruyama \\ Department of Sports and Health Science, Matsumoto University, Matsumoto, Japan \\ Email: fmaruya@nagoya-u.jp
}

How to cite this paper: Maruyama, F. (2020) Relationship between the Northern Hemisphere Sea Ice Area and Global Temperature by Multifractal Analysis. Journal of Applied Mathematics and Physics, 8, 896-909.

https://doi.org/10.4236/jamp.2020.85069

Received: April 17, 2020

Accepted: May 17, 2020

Published: May 20, 2020

Copyright $\odot 2020$ by author(s) and Scientific Research Publishing Inc. This work is licensed under the Creative Commons Attribution International License (CC BY 4.0).

http://creativecommons.org/licenses/by/4.0/

\section{(c) (i) Open Access}

\begin{abstract}
Changes in Arctic sea ice are an important fingerprint of natural and anthropogenic climate change. In general, fractal properties may be observed in the time series of the dynamics of complex systems. To study the relations among the Northern Hemisphere ( $\mathrm{NH})$ sea ice area, solar activity, global temperature, and Pacific Decadal Oscillation (PDO) index, we investigated changes in the fractal behavior of these time series by multifractal analysis and applying the wavelet coherence. The relationship between the solar activity: solar flux, sunspot number (SSN), and ultraviolet B (UV-B), and NH sea ice area was assessed based on changes in fractality. We identified the existing relations among the $\mathrm{NH}$ sea ice area, global temperature, and PDO based on changes in fractality and wavelet coherence. In the 2000s when the global warming hiatus occurred, after the order formation of $\mathrm{NH}$ sea ice area in the early 2000s, the change in state from multifractal to monofractal of the PDO and global temperature occurred, when fluctuations became large and multifractality became strong. We identified a small change in fractality for $\mathrm{NH}$ sea ice area, UV-B, and global temperature and a large change in fractality for solar flux, SSN, and PDO, which had large fluctuation. Our results will contribute to further studies on climate change.
\end{abstract}

\section{Keywords}

NH Sea Ice Area, Solar Flux, Global Temperature, PDO, Wavelet, Multifractal

\section{Introduction}

Changes in Arctic sea ice are an important fingerprint of natural and anthropogenic climate change. The dominant signal in sea ice variability over the satellite 
era (1979-present) is the reduction of sea ice extent, area, and thickness [1]. It has been suggested that the atmospheric circulation might be affected by sea ice [2]. Sea ice is a critical component of the climate system because it strongly influences albedo, surface turbulent heat fluxes, surface wind drag, and upper-ocean stratification. It is influenced by conditions in both the atmosphere and the ocean [3]. The sea-ice variability over the north Atlantic is linked to the NAO [4]. Variations in sea ice induce a greater atmospheric response associated with the NAO than those in global sea surface temperature [5]. Climate changes in the Northern Hemisphere have led to remarkable environmental changes in the Arctic Ocean [6]. Arctic sea-ice loss induced cooling anomalies over the Eurasian continent [7].

Various objects in nature show the so-called self-similarity or fractal property. Monofractal shows a roughly similar pattern at different scales and is characterized through a fractal dimension. Multifractal is a non-uniform, more complex fractal and is separated into many sub-sets characterized through different fractal dimensions. Fractal property can be observed in the time series representing dynamics of complex systems as well. A change of fractality occurs with a phase transition and changes of state. The multifractal properties of daily rain were studied in two contrasting climates: an East Asian monsoon climate with drastic rain variability and a mild climate with moderate rain variability [8]. In both the climates, the frontal rain shows monofractality and the convective-type rain shows multifractality.

Hence, climate change can be interpreted from the view of fractals. A change in fractality may be observed when the climate changes. We used the wavelet transform to analyze the multifractal behavior of the climate index. Wavelet methods are useful for the analysis of complex non-stationary time series. The wavelet transform allows good multifractal analysis to be performed [9]. We used the wavelet transform to analyze the multifractal behavior of the climate index. We concluded that a climatic regime shift corresponds to a change from multifractality to monofractality of the Pacific Decadal Oscillation (PDO) index [10] and showed the influence of solar activity on climatic regime shift [11].

To study the relations among the Northern Hemisphere (NH) sea ice area, solar activity, global temperature, and PDO, we investigated changes in the fractal behavior of these time series by multifractal analysis.

\section{Data and Method of Analysis}

We used the monthly sea ice index by National Snow and Ice Data Center (NSIDC) which shows NH sea ice area as shown in Figure 1. The solar radio flux at $10.7 \mathrm{~cm}$ (F10.7 flux) provided by NOAA's space weather prediction center (www.swpc.noaa.gov) was used. The F10.7 flux is an excellent indicator of the solar activity. The SSN provided by Solar Influences Data Analysis Center (sidc.oma.be) was used. The amount of UV-B is the value obtained by integrating the ultraviolet intensity of 280 to $315 \mathrm{~nm}$ in the wavelength range and we 
used the observations from Tsukuba (Japan) by the Japan Meteorological Agency. The global mean surface air temperature anomalies (Figure 2) and PDO index (Figure 3) provided by the NOAA's Climate Prediction Center, USA (CPC) were used.

For the examination, we used the Daubechies wavelet, which is widely used in

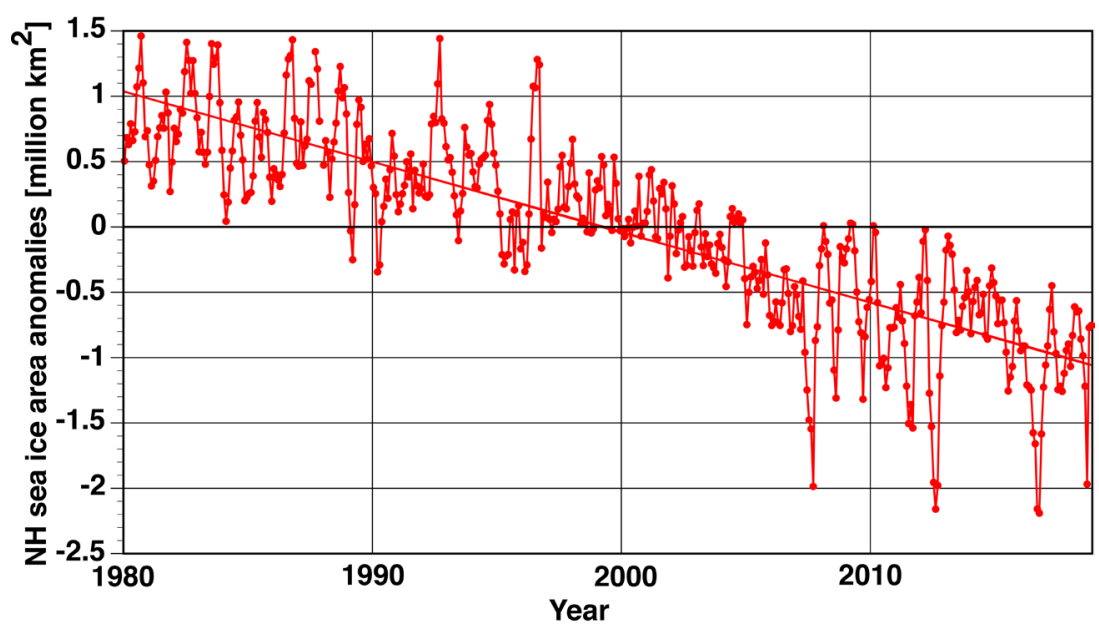

Figure 1. The time series of $\mathrm{NH}$ sea ice area anomalies.

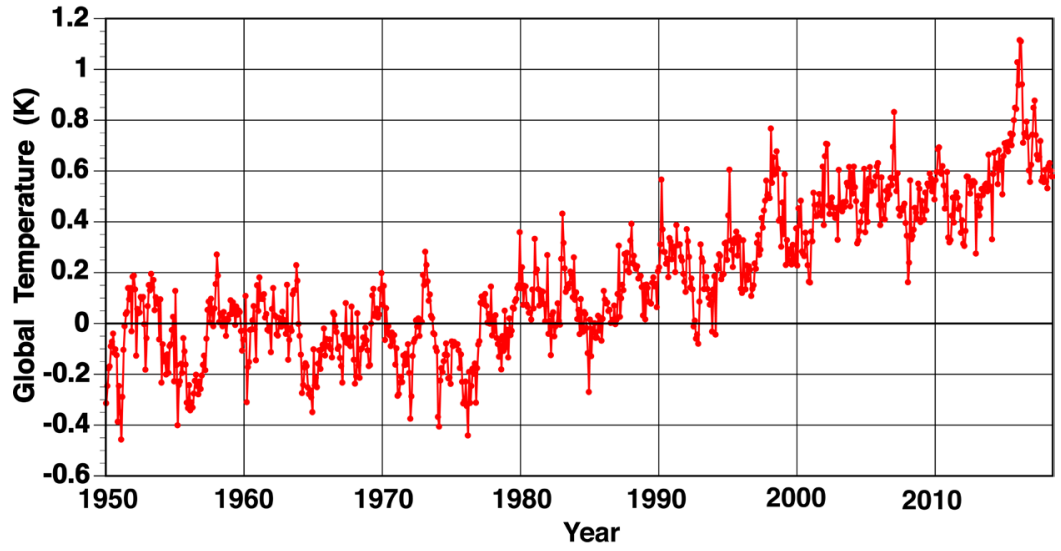

Figure 2. The time series of global mean surface air temperature anomalies.

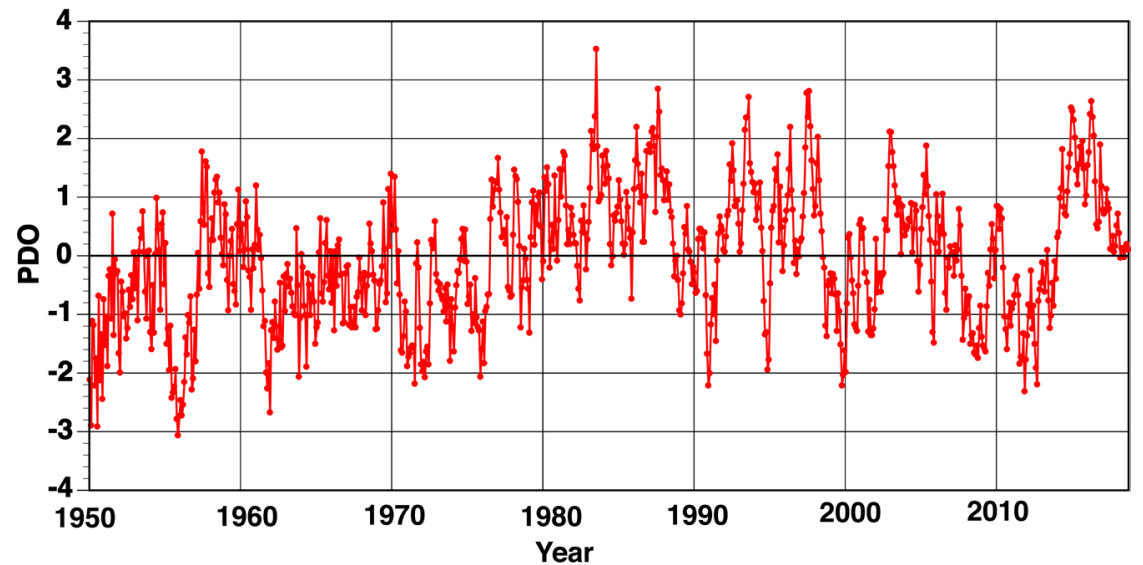

Figure 3. The time series of PDO index. 
solving a broad range of problems, e.g., self-similarity properties of a signal and signal discontinuities. We made use of a discrete signal that was fitted the Daubechies mother wavelet with the capacity of correct inverse transformation. Thus, we can precisely calculate the following best $\tau(q)$, which can be regarded as a characteristic function of the fractal behavior. We can define the $\tau(q)$ from the power-law behavior of the partition function, as shown in equation (3). We then computed the scaling of the partition function $Z_{q}(a)$, which is defined as the sum of the $q$-th powers of the modulus of the wavelet transform coefficients at scale a, where $q$ is the $q$-th moment. In our computation, the wavelet-transform coefficients did not grow zero. Thus, for a correct calculation, the summation was considered for the whole set. Muzy et al. [9] defined $Z_{q}(a)$ as the sum of the $q$-th powers of the local maxima of the modulus to avoid dividing by zero. We got the following partition function $Z_{q}(a)$ :

$$
Z_{q}(a)=\sum\left|W_{\varphi}[f](a, b)\right|^{q},
$$

where $W_{\varphi}[f](a, b)$, $a$, and $b$ are the wavelet coefficient of function $f$, a scale parameter, and a space parameter, respectively. $W_{\varphi}[f](a, b)$ is defined as below.

$$
W_{\phi}[f](a, b)=\frac{1}{\sqrt{|a|}} \int_{-\infty}^{+\infty} f(t) \phi^{*}\left(\frac{t-b}{a}\right) d t
$$

where $f(t)$ is data and $\phi$ is wavelet function. For small scales, we expect

$$
Z_{q}(a) \sim a^{\tau(q)} .
$$

First, we examined the changes in $Z_{q}(a)$ in the time series at a different scale $a$ for each moment $q$. We plotted the logarithm of $Z_{q}(a)$ against that of time scale a. Here $\tau(q)$ is the slope of the fitted straight line for each $q$. Next, we plotted $\tau(q)$ versus $q$. The time window was advanced by one year, which was repeated. The time window was fixed to 6 years, when a moderate change in fractality was observed. Monofractal and multifractal signals were defined as follows: For $\tau(q)$, a monofractal signal corresponds to a straight line, while a multifractal signal is nonlinear [12]. We calculated the $\mathrm{R}^{2}$ value, which is the coefficient of determination, for the fitted straight line. If $R^{2} \geq 0.98$, the time series is monofractal and if $0.98>\mathrm{R}^{2}$, it is multifractal.

We calculated $\tau(q)$ for moments $q=-6,-5,-4,-3,-2,-1,0,1,2,3,4,5,6$ for individual records for the SSN. In Figure 4, $\pi(q)$ for individual SSN from 1967 to 1979 is shown. The data was analyzed in 6-year sets. For instance, $\tau(q)$ of s70 was calculated from 1970 to 1975 , and that of s71 was calculated from 1971 to 1976 . To detect changes in fractality, the time window was then shifted forward one year and $\tau(q)$ was calculated from s67 to s76. A monofractal signal corresponds to a straight line for $\tau(q)$, whereas for a multifractal signal, $\tau(q)$ is nonlinear. In Figure 4, $\tau(q)$ is linear for $\mathrm{s} 69$ - $\mathrm{s} 71$, which indicates monofractality. In contrast, the nonlinear $\tau(q)$ curves for s67, s68, and s72 - s74 show multifractality.

We plotted the value of $t(-6)$ in each index. The negative large value of $t(-6)$ 


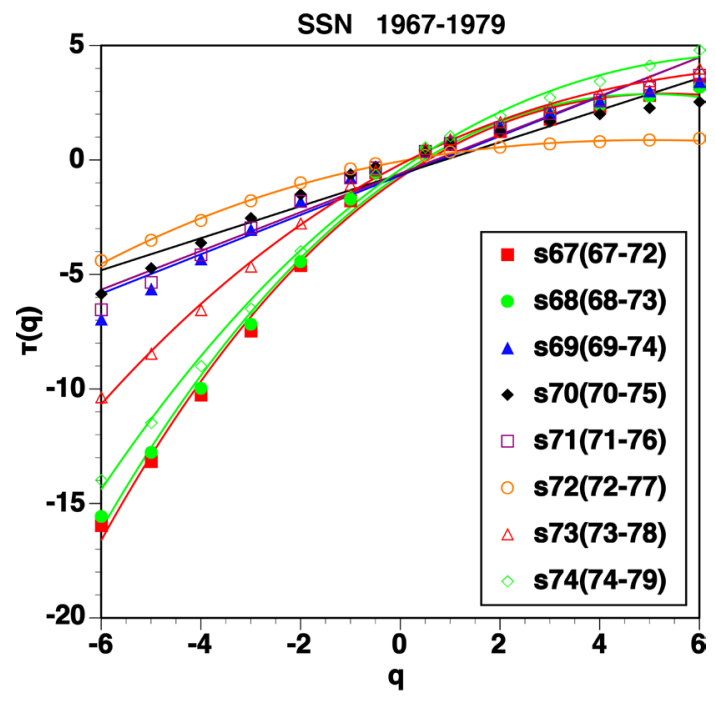

Figure 4. $\tau(q)$ for individual SSN between 1967 and 1979.

shows large multifractality. For $\tau(\mathrm{q}), q=-6$ is the appropriate number to show the change of $\tau$.

\section{Results}

\subsection{The Relationship between the Solar Activity and NH Sea Ice Area}

The time series of NH sea ice area anomalies is shown in Figure 1. The NH sea ice area has decreased since 1980. For 1997-2005, the decrease was small and for 2008-2018 the decrease was large in some years. Decrease amount increased after the 2000s.

The $\tau(-6)$ of F10.7 flux and NH sea ice area are shown in Figure 5. The red square shows monofractality and the green circle shows multifractality for the 6 years centered on the year plotted. For instance, the green circle in 1980 for the F10.7 flux shows multifractality between 1977 and 1982. The data was excluded from Figure 5 for cases where we could not distinguish between monofractality and multifractality. The changes in fractality of F10.7 flux were larger than that of $\mathrm{NH}$ sea ice area. The changes in fractality of $\mathrm{NH}$ sea ice area and F10.7 flux were very similar in the 2000 s.

The $\tau(-6)$ density plot of NH sea ice area and F10.7 flux are shown in Figure 6(a) and Figure 6(b), respectively. The NH sea ice area showed narrow density distribution with single peak, indicating a small change in fractality. In contrast, F10.7 flux showed wide density distribution with two peaks and strong multifractality, indicating a large change in fractality.

The $\tau(-6)$ of SSN and NH sea ice area are shown in Figure 7. The change in fractality of SSN was larger than that of NH sea ice area. The changes in fractality of SSN and NH sea ice area were very similar in the 2000s.

The emitted solar energy and ultraviolet rays change very similarly, so ultraviolet rays represent solar energy. The $\tau(-6)$ of UV-B and NH sea ice area are 
shown in Figure 8. The changes in fractality of UV-B and NH sea ice area were very similar in the 2000s.

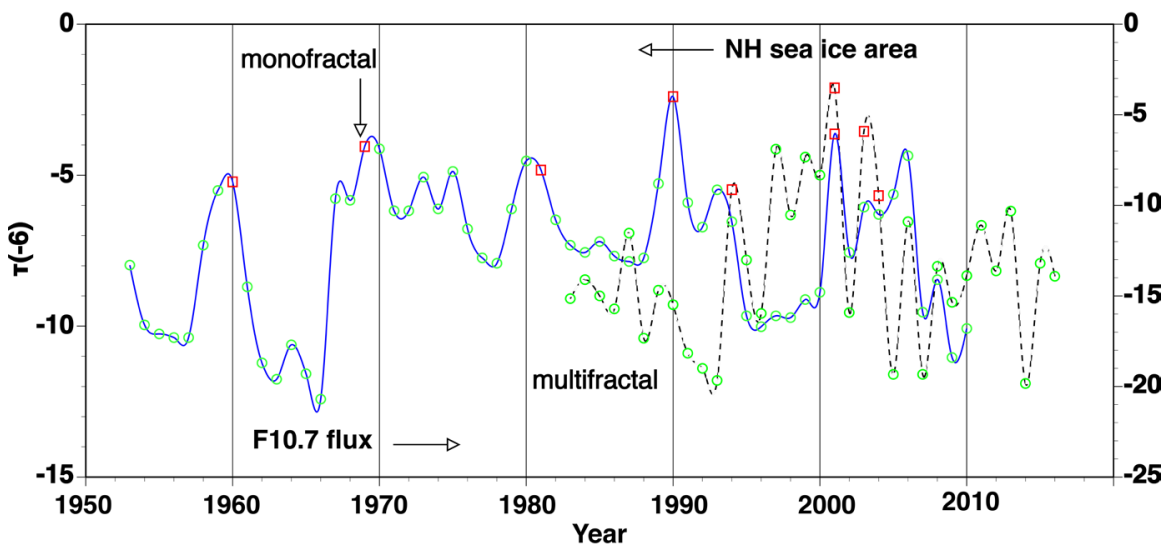

Figure 5. The $t(-6)$ of F10.7 flux and NH sea ice area.
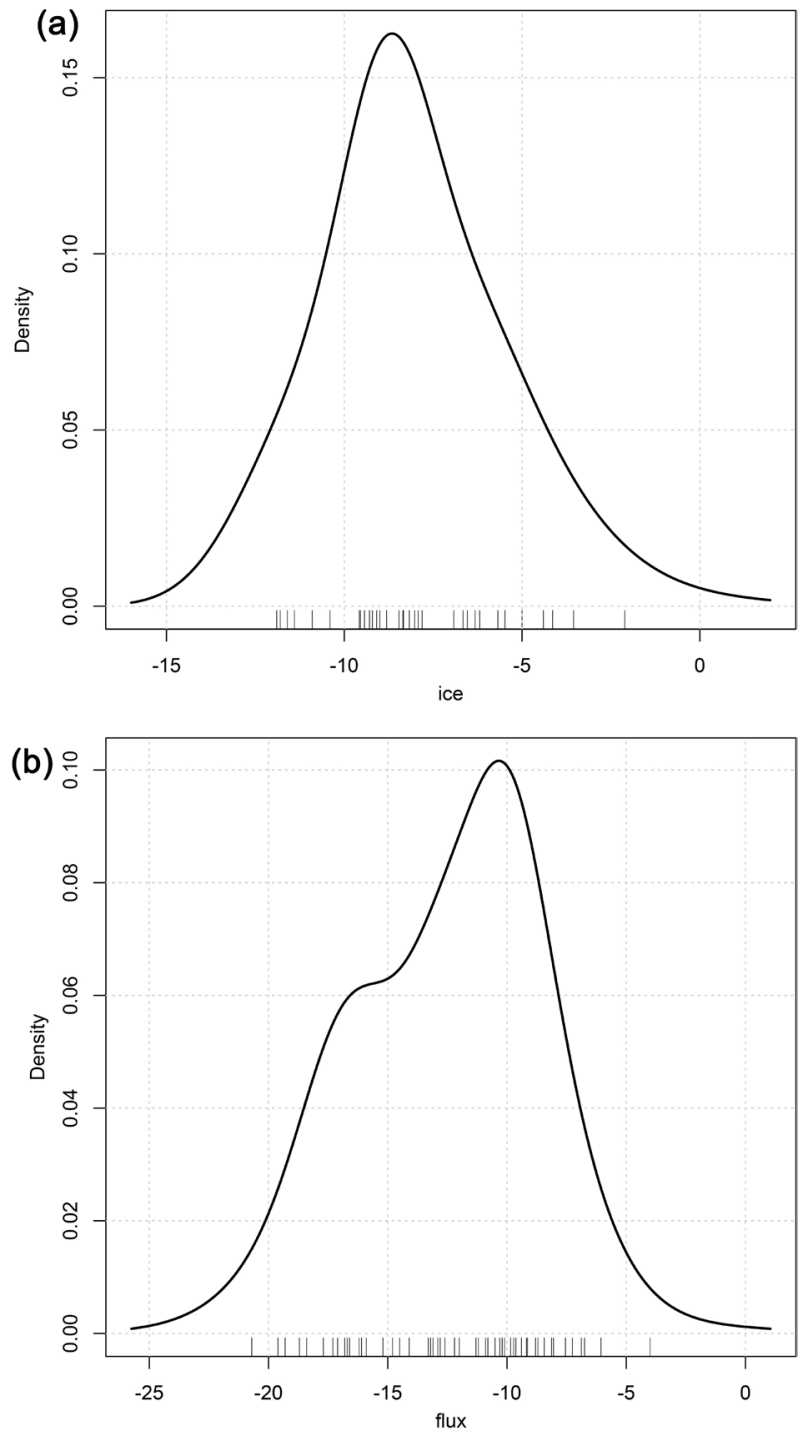

Figure 6. The $t(-6)$ density plot of NH sea ice area (a) and F10.7 flux (b). 


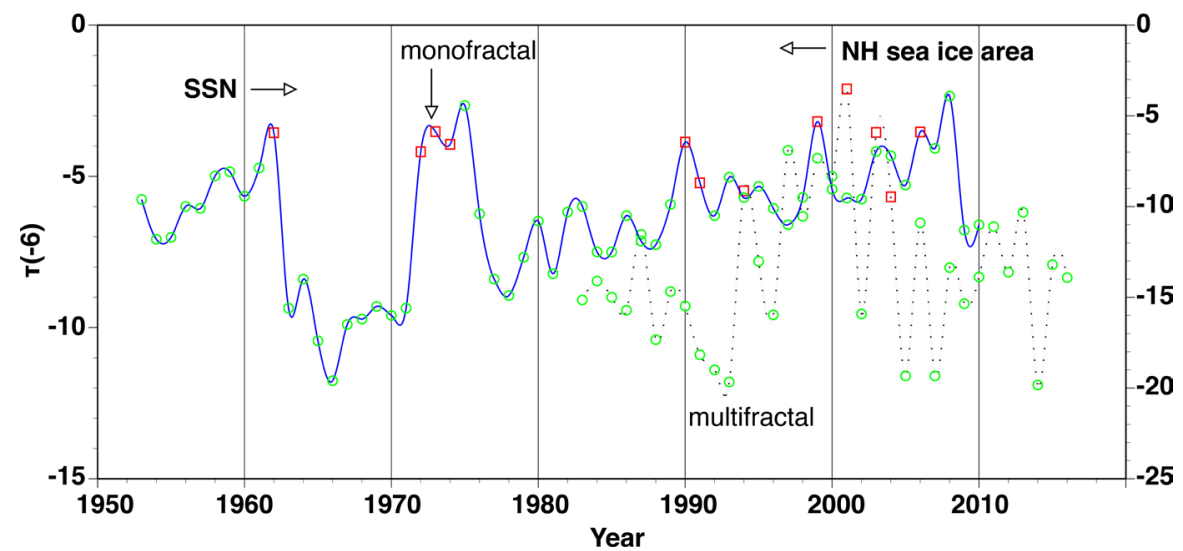

Figure 7. The $\tau(-6)$ of SSN, and NH sea ice area.

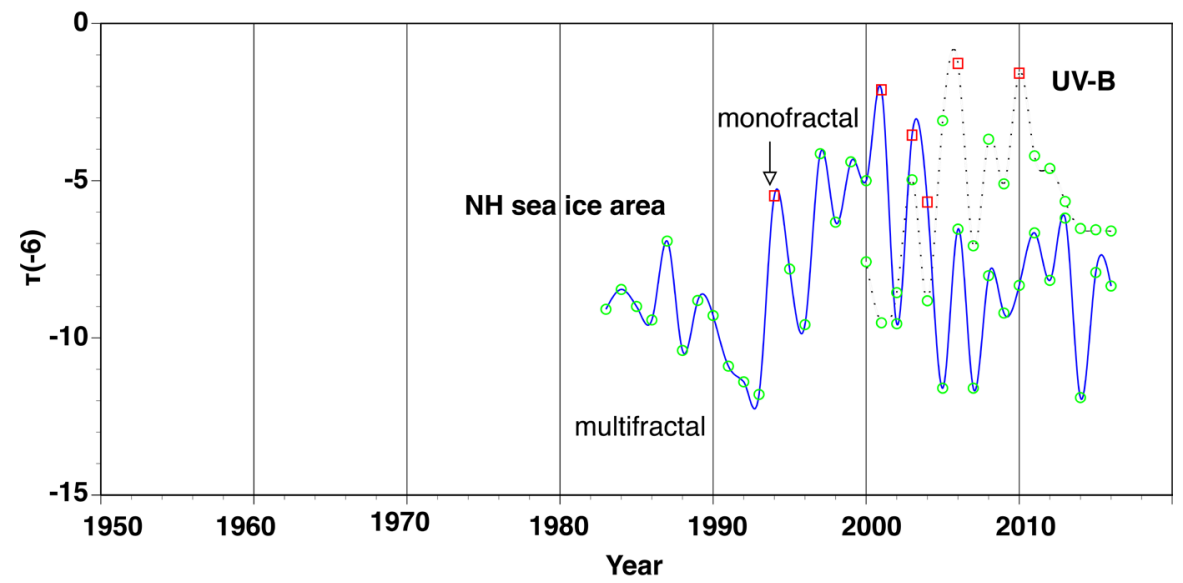

Figure 8. The $\tau(-6)$ of UV-B, and NH sea ice area.

\subsection{The Relationship between the Global Temperature and NH Sea Ice Area}

The time series of $\mathrm{NH}$ sea ice area anomalies and global mean surface air temperature anomalies are shown in Figure 1 and Figure 2, respectively. For 1998-2005 the decrease of NH sea ice area was small, which corresponded to the global warming hiatus for 1998-2012 as shown in Figure 2. The rate of global mean surface temperature increase slowed between 1998 and 2012 and the change was often termed the "global warming hiatus" [13]. The minimum of NH sea ice area for 2008-2018 corresponded to the maximum of global temperature as shown in Figure 2. The $\tau(-6)$ of global temperature and $\mathrm{NH}$ sea ice area are shown in Figure 9(a). The global temperature significantly led $\mathrm{NH}$ sea ice area from the cross-correlation functions (CCF). The changes in fractality of global temperature and $\mathrm{NH}$ sea ice area were consistent in some places.

We studied the relationship between the global temperature and $\mathrm{NH}$ sea ice area by means of wavelet coherence. We show the wavelet coherence and phase using the Morlet wavelet between the global temperature and $\mathrm{NH}$ sea ice area in Figure 9(b). The coherence between the global temperature and $\mathrm{NH}$ sea ice area in one year scale was strong, and the lead of $\mathrm{NH}$ sea ice area was observed. 


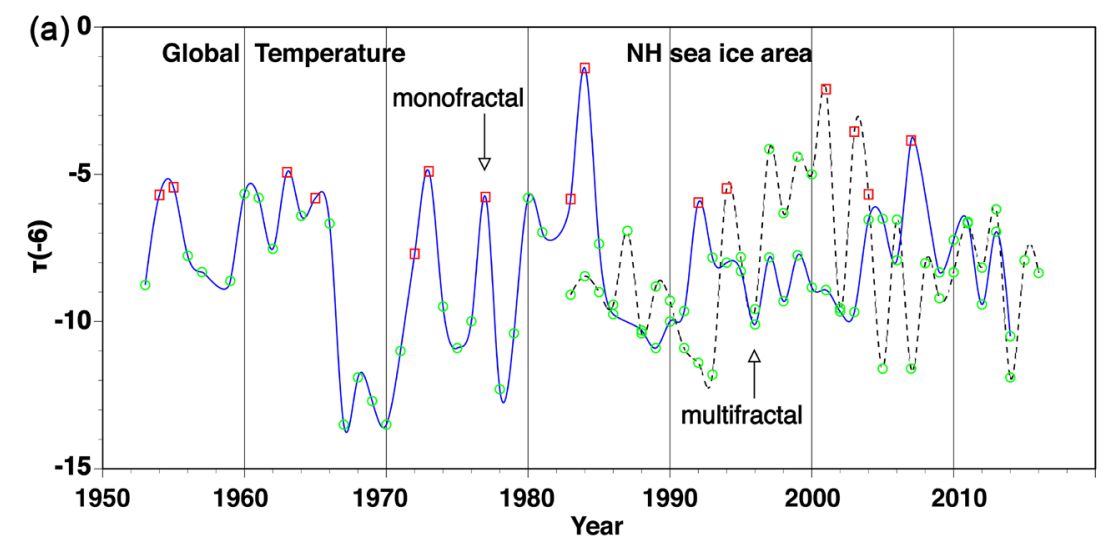

(b)

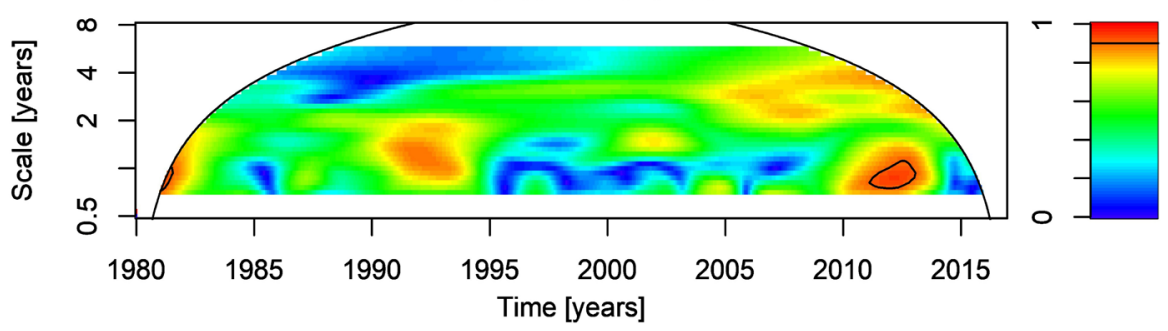

Phase

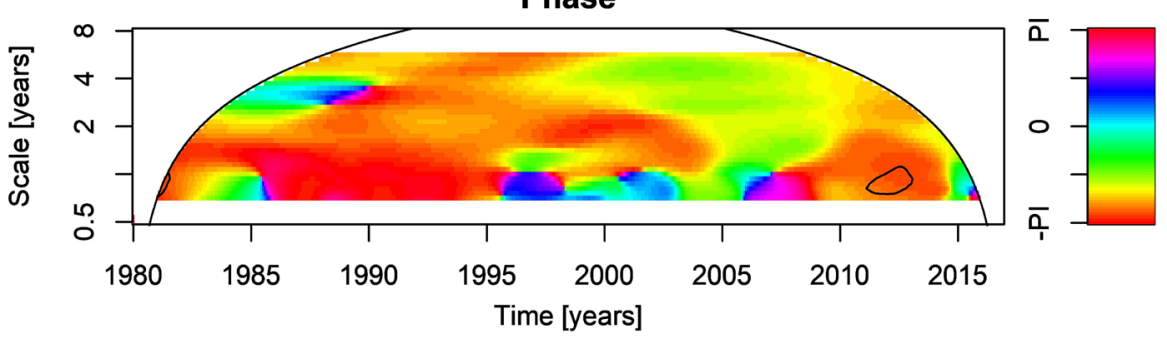

Figure 9. The $t(-6)$ of global temperature, and $\mathrm{NH}$ sea ice area (a). Wavelet coherence and phase between global temperature and $\mathrm{NH}$ sea ice area (b). In the wavelet phase, the positive value shown by the blue and pink shading means that global temperature leads $\mathrm{NH}$ sea ice area and the negative value shown by the green, yellow and red shading means that $\mathrm{NH}$ sea ice area leads global temperature.

\subsection{The Relationship between the PDO and NH Sea Ice Area}

The time series of the PDO index is shown in Figure 3. The minimum of the $\mathrm{NH}$ sea ice area in 2018 corresponded to the maximum of the PDO. The $t(-6)$ of PDO and NH sea ice area are shown in Figure 10(a). The PDO significantly led $\mathrm{NH}$ sea ice area from the cross-correlation functions (CCF). The change in fractality of PDO was larger than that of NH sea ice area. For the period describing the global warming hiatus, the changes in fractality were very similar in the 2000s.

We studied the relationship between the PDO and $\mathrm{NH}$ sea ice area by means of wavelet coherence. We show the wavelet coherence and phase using the Morlet wavelet between the PDO and NH sea ice area in Figure 10(b). The coherence between the PDO and $\mathrm{NH}$ sea ice area was strong, and the lead of the PDO was observed. 

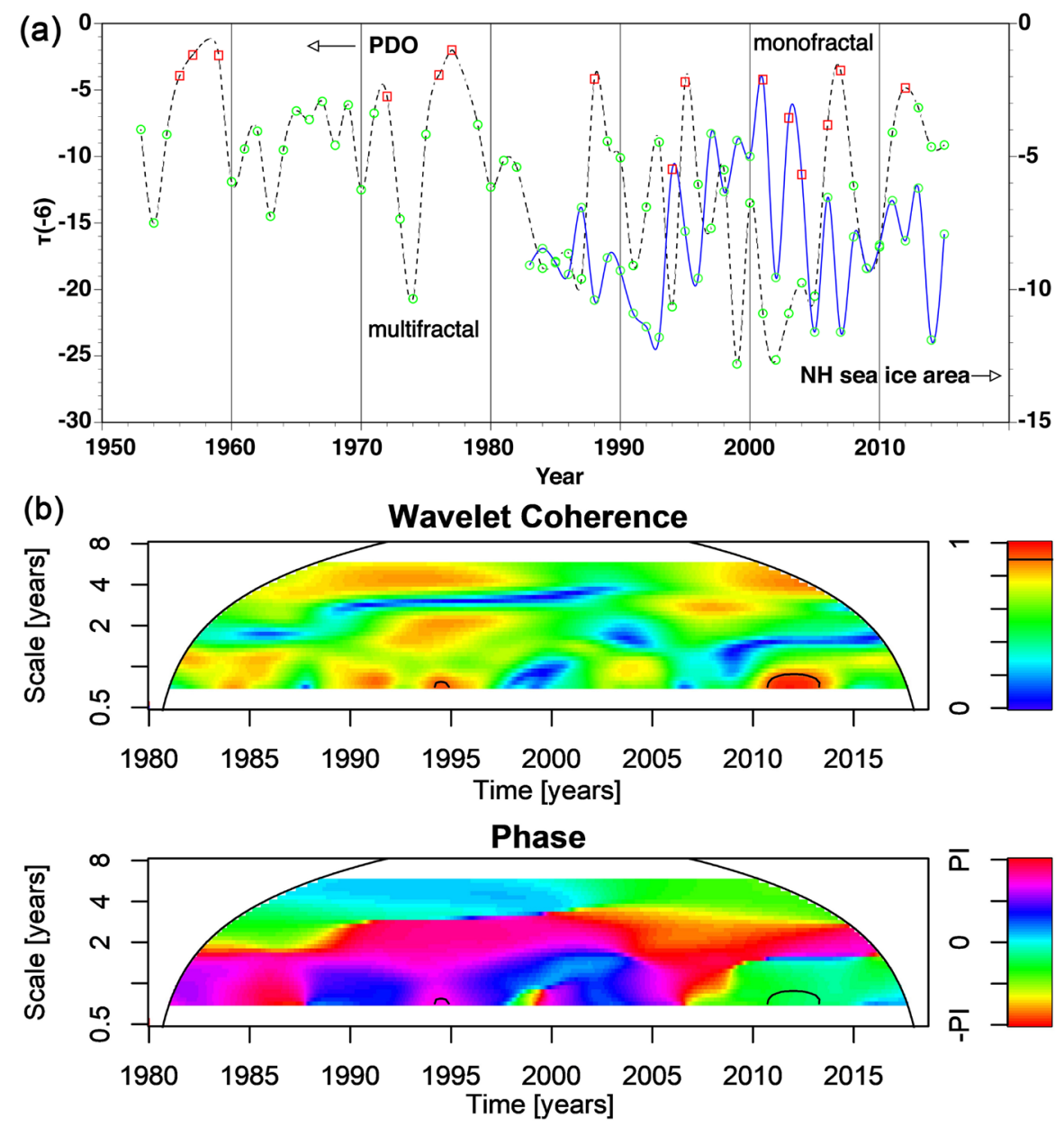

Figure 10. The $t(-6)$ of PDO, and NH sea ice area (a). Wavelet coherence and phase between PDO and NH sea ice area (b). In the wavelet phase, the positive value shown by the blue and pink shading means that PDO leads NH sea ice area and the negative value shown by the green, yellow and red shading means that NH sea ice area leads PDO.

\subsection{The Relationship between the PDO and Global Temperature}

When the global warming hiatus occurred for 1998-2012, the PDO was negative mainly. The $t(-6)$ of PDO and global temperature are shown in Figure 11(a). We calculated the autocorrelation function (ACF) of $t(-6)$, which showed 5 -year periodicity. The PDO significantly led global temperature from the cross-correlation functions (CCF). The change in fractality of PDO was larger than that of global temperature. Around 2007 the PDO and global temperature have peaks, and have monofractality. Fractality for PDO and global temperature changed with many reverse changes. For the period describing the global warming hiatus, the changes in fractality were very similar in the 2000s. Figure 11(b) shows the wavelet coherence and phase between the PDO and global temperature using the Morlet wavelet. The coherence between the PDO index and global temperature in the 2-year scale was strong for 1970-1985, and the lead of the PDO index was observed.

The $t(-6)$ density plot of global temperature and PDO are shown in Figure 12(a) 

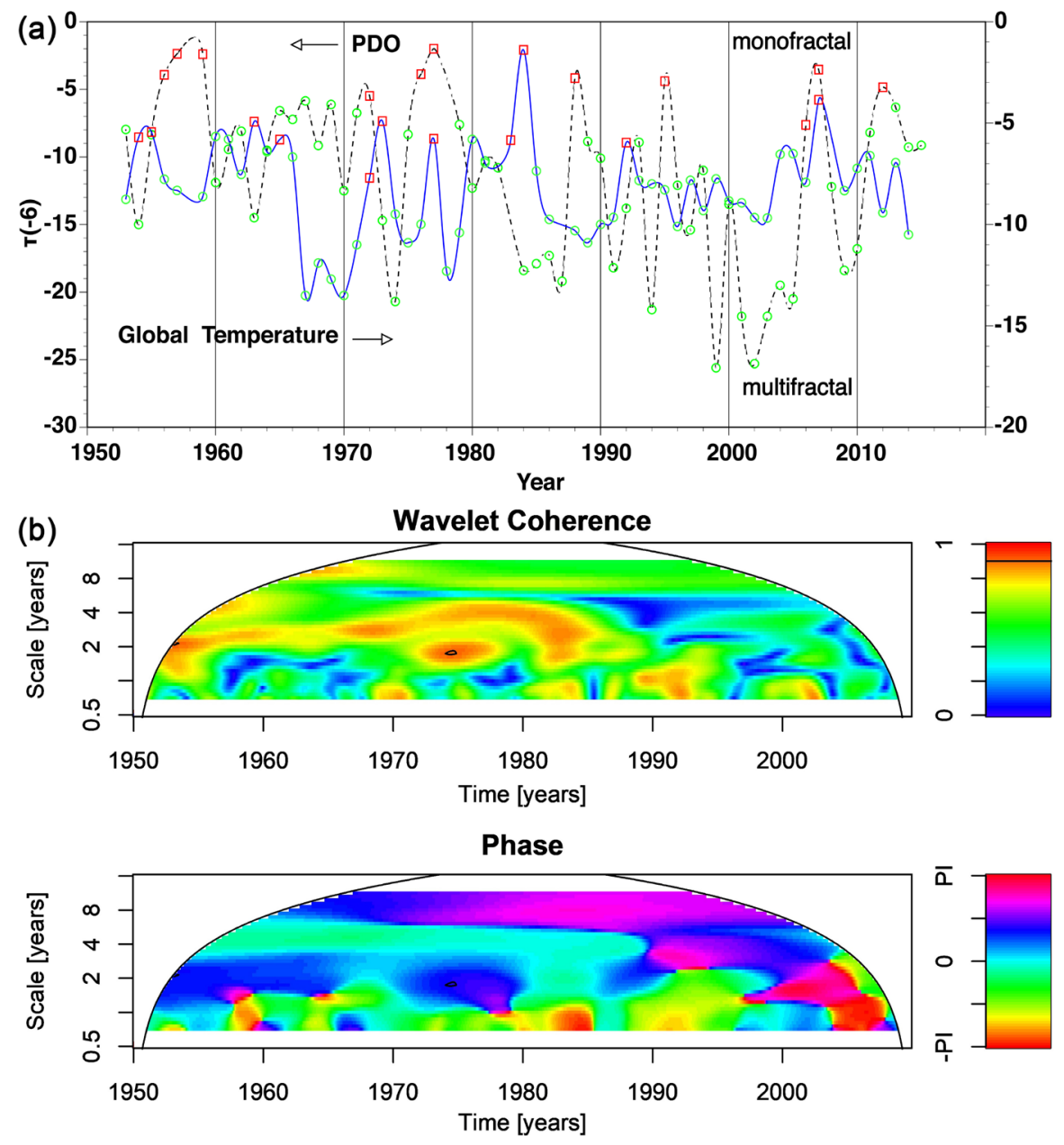

Figure 11. The $t(-6)$ of PDO, and global temperature (a). Wavelet coherence and phase between PDO and global temperature (b). In the wavelet phase, the positive value shown by the blue and pink shading means that PDO leads global temperature and the negative value shown by the green, yellow and red shading means that global temperature leads PDO.

and Figure 12(b), respectively. The global temperature showed narrow density distribution with single peak and the distribution was narrow, indicating a small change in fractality. In contrast, the PDO showed wide density distribution with two peaks and strong multifractality, indicating a large change in fractality, then the change from multifractal to monofractal was observed. In 1976 the fractality changed from multifractality to monofractality for the PDO, which was climate regime shift.

\section{Discussion}

We found that the time series of NH sea ice area, solar flux, SSN, UV-B, global temperature, and PDO had fractal properties by multifractal analysis. The changes in fractality of $\mathrm{NH}$ sea ice area and solar activity (F10.7 flux, SSN, UV-B) were very similar in the 2000s, which showed the influence of solar activity on NH sea ice area. In the early 2000 s, the $\mathrm{NH}$ sea ice area showed monofractality and the 

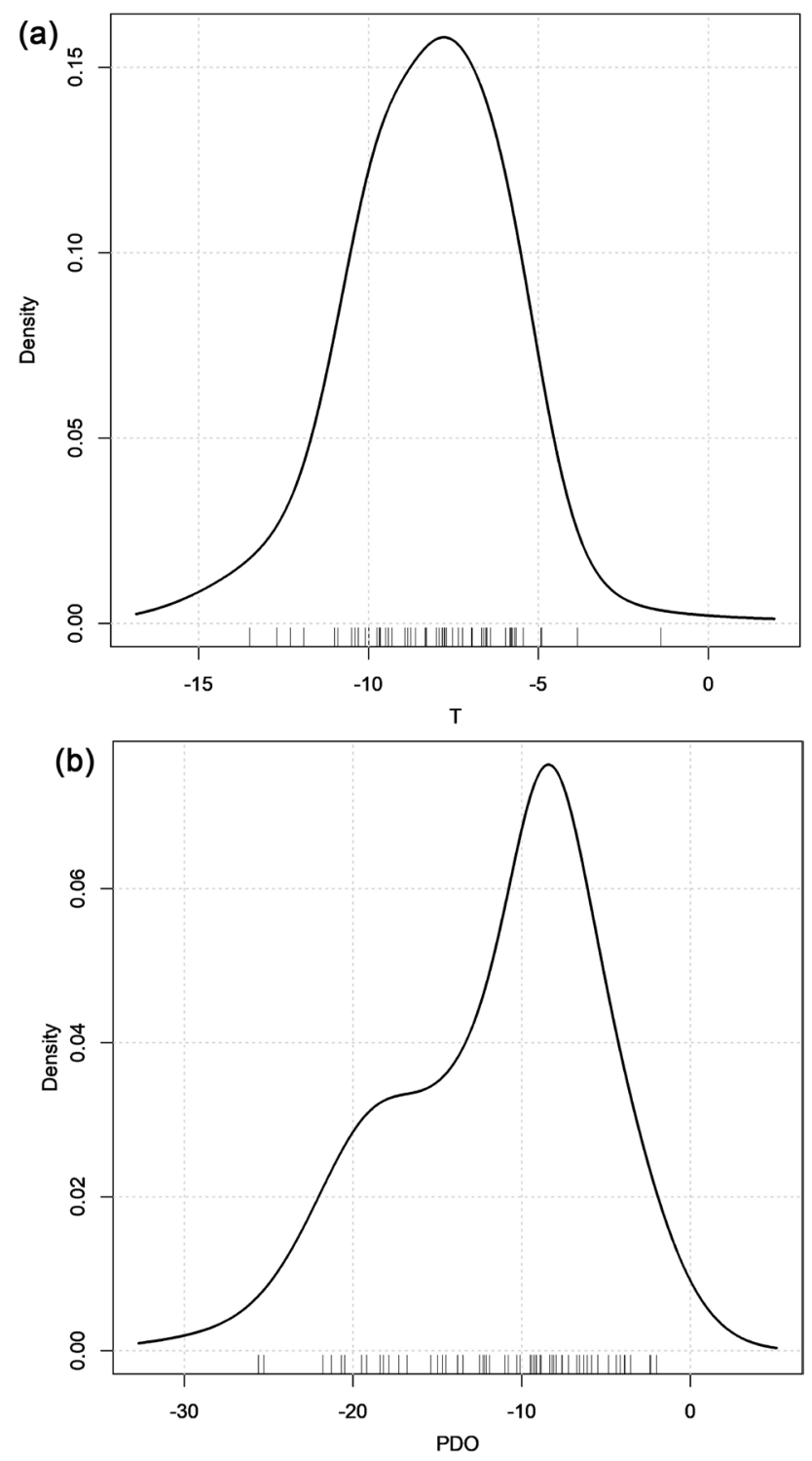

Figure 12. The $t(-6)$ density plot of global temperature (a) and PDO (b).

order formation, then became multifractality and fluctuation increased. The F10.7 flux also showed monofractality in 2001, when the SSN was maximum and the solar activity was active. Hence the relationship between the solar activity and $\mathrm{NH}$ sea ice area was observed from the view of fractal.

The coherence between the PDO and NH sea ice area was strong and changes in fractality between the PDO and $\mathrm{NH}$ sea ice area were similar. The lead of the PDO to NH sea ice area was observed by changes in fractality and wavelet analysis. The PDO directly determine the ocean temperature of the Pacific sectors of the Arctic. The sea ice concentrate on is reduced in the warm phase, which can be partially attributed to the recent Arctic sea ice retreat [14]. The lead of the PDO to global temperature was observed by changes in fractality and wavelet analysis. During the period of the global warming hiatus, the changes in fractality between the PDO and global temperature were very similar in the 2000s. The 
influences of the PDO on NH sea ice area and global temperature were assessed based on changes in fractality and wavelet coherence. Sea ice is a sensitive component of the climate system, and influenced by the conditions in both the atmosphere and ocean [3].

It has been suggested that the atmospheric circulation might be affected by sea ice [2]. Sea ice strongly influences albedo, surface turbulent heat fluxes, surface wind drag, and upper-ocean stratification, hence sea ice is a critical component of the climate system. The NH sea ice area was found to lead global temperature according to the wavelet analysis. We identified a relationship between the $\mathrm{NH}$ sea ice area and global temperature: higher global temperatures reduced sea ice and sunlight reflection and increased solar heat absorption, which in turn increased global temperature. The influence of global temperature on sea ice area was assessed based on changes in fractality, while the influence of sea ice area on global temperature was assessed based on wavelet coherence. The $\mathrm{NH}$ sea ice area showed monofractality and the order formation in the early 2000s. In the mid 2000s, the PDO and global temperature showed the change in state from multifractal to monofractal. When fluctuations became large and multifractality became strong, a change from multifractal to monofractal behavior was observed. Hence, in the 2000s when the global warming hiatus occurred, after the order formation of $\mathrm{NH}$ sea ice area, the change in state of the PDO and global temperature occurred. The relationship between the $\mathrm{NH}$ sea ice area and global temperature was shown from the changes in fractality.

The NH sea ice area, UV-B, and global temperature showed narrow density distributions with single peak, indicating a small change in fractality. In contrast, the F10.7 flux, SSN, and PDO showed wide density distributions with two peaks and strong multifractality, large fluctuation, indicating a large change in fractality.

\section{Conclusions}

To study the relations among the NH sea ice area, solar activity, global temperature, and PDO, we investigated the changes in the fractal behavior of these time series using multifractal analysis. Changes in fractality were detected by plotting the $\tau$-function and applying the wavelet coherence. Our results are summarized as follows:

1) The relationship between the solar activity (F10.7 flux, SSN, UV-B) and NH sea ice area was assessed based on changes in fractality.

2) We identified the existing relations among the $\mathrm{NH}$ sea ice area, global temperature, and PDO based on changes in fractality and wavelet coherence. In the 2000s when the global warming hiatus occurred, after the order formation of $\mathrm{NH}$ sea ice area in the early 2000s, the change in state from multifractal to monofractal of the PDO and global temperature occurred, when fluctuations became large and multifractality became strong.

3) We identified a small change in fractality for NH sea ice area, UV-B, and global temperature and a large change in fractality for F10.7 flux, SSN, and PDO, 
which had large fluctuation.

\section{Conflicts of Interest}

The author declares no conflicts of interest regarding the publication of this paper.

\section{References}

[1] Schweiger, A.J., Wood, K.R. and Zhang, J. (2019) Arctic Sea Ice Volume Variability over 1901-2010: A Model-Based Reconstruction. Journal of Climate, 32, 4731-4752. https://doi.org/10.1175/jcli-d-19-0008.1

[2] Alexander, M.A., Bhatt, U.S., Walsh, J.E., Timlin, M.S., Miller, J.S. and Scott, J.D. (2004) The Atmospheric Response to Realistic Arctic Sea Ice Anomalies in an AGCM during Winter. Journal of Climate, 17, 890-905. https://doi.org/10.1175/1520-0442(2004)017<0890:tartra $>2.0 . c 0 ; 2$

[3] Deser, C., Walsh, J.E. and Timlin, M.S. (2000) Arctic Sea Ice Variability in the Context of Recent Atmospheric Circulation Trends. Journal of Climate, 13, 617-633. https://doi.org/10.1175/1520-0442(2000)013<0617:asivit>2.0.co;2

[4] Rogers, J.C. and Loon, H.V. (1978) The Seasaw in Winter Temperatures between Greenland and Northern Europe. Part 2: Some Oceanic and Atmospheric Effects in Middle and High Latitudes. Monthly Weather Review, 107, 509-519. https://doi.org/10.1175/1520-0493(1979)107<0509:tsiwtb>2.0.co;2

[5] Magnusdottir, G., Deser, C. and Saravanan, R. (2004) The Effects of North Atlantic SST and Sea Ice Anomalies on the Winter Circulation in CCM3. Part 1: Main Features and Storm Track Characteristics of the Response. Journal of Climate, 17, 857-876. https://doi.org/10.1175/1520-0442(2004)017<0857:teonas >2.0.co;2

[6] Semiletov, I., Makshtas, A. and Akasofu, S. (2004) Atmospheric $\mathrm{CO}_{2}$ Balance: The Role of Arctic Sea Ice. Geophysical Research Letters, 31, L05121. https://doi.org/10.1029/2003gl017996

[7] Nakamura, T., Yamazaki, K., Sato, T. and Ukita, J. (2019) Memory Effects of Eurasian Land Processes Cause Enhanced Cooling in Response to Sea Ice Loss. Nature Communications, 10, Article ID: 5111. https://doi.org/10.1038/s41467-019-13124-2

[8] Svensson, C., Olsson, J. and Berndtsson, R. (1996) Multifractal Properties of Daily Rainfall in Two Different Climates. Water Resources Research, 32, 2463-2472. https://doi.org/10.1029/96wr01099

[9] Muzy, J.F., Bacry, E. and Arneodo, A. (1991) Wavelets and Multifractal Formalism for Singular Signals: Application to Turbulence Data. Physical Review Letters, 67, 3515-3518. https://doi.org/10.1103/physrevlett.67.3515

[10] Maruyama, F., Kai, K. and Morimoto, H. (2015) Wavelet-Based Multifractal Analysis on Climatic Regime Shifts. Journal of the Meteorological Society of Japan, 93, 331-341. https://doi.org/10.2151/jmsj.2015-018

[11] Maruyama, F., Kai, K. and Morimoto, H. (2017) Wavelet-Based Multifractal Analysis on a Time Series of Solar Activity and PDO Climate Index. Advances in Space Research, 60, 1363-1372. https://doi.org/10.1016/j.asr.2017.06.004

[12] Frish, U. and Parisi, G. (1985) On the Singularity Structure of Fully Developed Turbulence, in Turbulence and Predictability. In: Ghil, M., Benzi, R. and Parisi, G., Eds., Geophysical Fluid Dynamics and Climate Dynamics, North-Holland, New York, 84-88. 
[13] Medhang, I., Stolpe, M.B., Fischer, E.M. and Knutti, R. (2017) Reconciling Controversies about the 'Global Warming Hiatus'. Nature, 545, 41-47. https://doi.org/10.1038/nature22315

[14] Shimada, K., Kamoshida, T., Itoh, M., Nishino, S., Carmac, E., McLaughlin, F., Zimmermann, S. and Proshutinsky, A. (2006) Pacific Ocean Inflow: Influence on Catastrophic Reduction of Sea Ice Cover in the Arctic Ocean. Geophysical Research Letters, 33, L08605. https://doi.org/10.1029/2005gl025624 